\title{
THE ISOMORPHISM QUESTION FOR MODULAR GROUP ALGEBRAS OF METACYCLIC $p$-GROUPS
}

\author{
CZESŁAW BAGIŃSKI
}

(Communicated by Donald S. Passman)

\begin{abstract}
Let $F[G]$ be a group algebra of a finite $p$-group $G$ over the field $F=G F(p)$. If $G \simeq H$, then clearly $F[G] \simeq F[H]$. However, it is not known whether the converse is true. The answer for metacyclic $p$-groups, $p>3$, is given.
\end{abstract}

Let $p$ be a prime, $G$-a finite nonabelian $p$-group and $F=G F(p)$-the field with $p$ elements. In this note we study the following isomorphism problem: Whether the isomorphism of group algebras $F[G]$ and $F[H]$ implies the isomorphism of groups $G$ and $H$. In [4] it was given the positive answer to this question for all $p$-groups of order at most $p^{4}$. Here we prove the following

THEOREM. If $G$ is a metacyclic $p$-group, $p>3$, and $F[G] \simeq F[H]$, then $G \simeq H$.

Our result depends on some lemmas. We use standard notation, see [3, 5].

LEMMA 1. Let $N$ be a normal subgroup of a finite p-group $G$. If $I=$ $\omega(F[N]) F[G]$, then $I^{n}=\omega(F[N])^{n} F[G]$. Moreover, if $\eta_{i}, i=1, \ldots, t$, form $a$ basis of the space $\omega(F[N])^{n}$ and $\left\{1=g_{1}, \ldots, g_{s}\right\}$ is a right transversal for $N$ in $G$, then $\eta_{i} g_{j}, i=1, \ldots, t, j=1, \ldots, s$, form a basis of the space $I^{n}$.

The proof of the first part is by an easy induction on $n$. The second part is obvious.

For any finite $p$-group $G$ let $\left\{\mathscr{M}_{i}(G)\right\}$ be the Brauer-Jennings-Zassenhaus $\mathscr{M}$ series of a $p$-group $G$ defined by $\mathscr{M}_{1}(G)=G$ and for $n \geq 2$

$$
\mathscr{M}_{n}(G)=\left(\mathscr{M}_{n-1}(G), G\right) \mathscr{M}_{i}(G)^{(p)},
$$

where $i$ is the smallest integer satisfying $i p \geq n$.

LEMMA 2. If $G^{\prime}$ is the commutator subgroup of $G$, then the factor groups $\mathscr{M}_{i}\left(G^{\prime}\right) / \mathscr{M}_{i+1}\left(G^{\prime}\right)$ for all $i$ are determined by $F[G]$.

PROOF. By proof of $[5,14.2 .7 \mathrm{i}]$ the factor groups $\mathscr{M}_{i}\left(G^{\prime}\right) / \mathscr{M}_{i+1}\left(G^{\prime}\right)$ are determined up to isomorphism by

$$
\begin{aligned}
f_{j} & =\operatorname{dim}_{F} \omega\left(F\left[G^{\prime}\right]\right)^{j} / \omega\left(F\left[G^{\prime}\right]\right)^{j+1} \\
& =\frac{1}{\left|G: G^{\prime}\right|} \operatorname{dim}_{F} \omega\left(F\left[G^{\prime}\right]\right)^{j} F[G] / \omega\left(F\left[G^{\prime}\right]\right)^{j+1} F[G] .
\end{aligned}
$$

Since $\omega\left(F\left[G^{\prime}\right]\right) F[G]$ as an ideal generated by the subspace $[F[G], F[G]]$, is determined by $F[G]$ and $\left|G: G^{\prime}\right|=\operatorname{dim}_{F} F[G] / \omega\left(F\left[G^{\prime}\right]\right) F[G]$ the result follows from Lemma 1.

Received by the editors October 30, 1986 and, in revised form, August 27, 1987.

1980 Mathematics Subject Classification (1985 Revision). Primary 16A26. 
By the proof of $[5,14.2 .7 \mathrm{ii}]$ we obtain then

COROLlARY 1. If $G^{\prime}$ is cyclic, then the isomorphism class of $G^{\prime}$ is determined by $F[G]$. In particular, if $F[G] \simeq F[H]$ and $G^{\prime}$ is cyclic, then $H^{\prime}$ is cyclic too.

Remark now that if $G$ is a metacyclic $p$-group, then by the inclusion $\left(\mathscr{M}_{n}(G), G\right)$ $\subset \mathscr{M}_{n}(G)^{(p)}$ we have $\mathscr{M}_{2}(G)=G^{(p)}=\mathscr{M}_{3}(G)=\cdots=\mathscr{M}_{p}(G), \mathscr{M}_{p+1}(G)=\cdots=$ $\mathscr{M}_{p^{2}}(G)=G^{\left(p^{2}\right)}$ and so on. Thus we have

COROLlaRY 2. If $G$ is a metacyclic p-group then the exponent of $G$ is determined by $F[G]$.

LEMMA 3. If $G$ is a finite p-group with $G^{\prime}$ cyclic, then for every integer $n \geq 1$

$$
\omega\left(F\left[G^{\left(p^{n}\right)}\right]\right) F[G]=\left(\omega\left(F\left[G^{\prime}\right]\right) F[G]\right)^{p^{n}} .
$$

Proof. Let $G^{\prime}=\langle g\rangle$. Elements $g-1,(g-1)^{2}, \ldots,(g-1)^{o(g)-1}$ form a basis of $\omega\left(F\left[G^{\prime}\right]\right)[\mathbf{5}, 3.3 .3]$. So $\omega\left(F\left[G^{\prime}\right]\right)^{p^{n}} \subset \omega\left(F\left[G^{\prime\left(p^{n}\right)}\right]\right) F[G] \subset \omega\left(F\left[G^{\prime}\right]\right)^{p^{n}} F[G]$ and then by Lemma $1 \omega\left(F\left[G^{\prime\left(p^{n}\right)}\right]\right) F[G]=\left(\omega\left(F\left[G^{\prime}\right]\right) F[G]\right)^{p^{n}}$.

Let now $\Omega_{1}(G)=\left\langle x \in G \mid x^{p}=1\right\rangle$. We shall say that $G$ is a $p_{1}$-group iff $x^{p}=y^{p}$ implies $\left(x y^{-1}\right)^{p}=1$ for all $x, y \in G$. Let $\omega_{1}(F[G])$ be the ideal of $F[G]$ generated by all elements $a \in F[G]$ satisfying $a^{p}=0$.

LEMMA 4 [1, LEMMA 3]. If $G$ is a $p_{1}$-group and for all $g \in G g^{p} \in Z(G)$, then $\omega_{1}(F[G])=\omega(F[H]) F[G]$, where $H=\Omega_{1}(G)$.

PROOF OF THE THEOREM. Let $F[G] \simeq F[H]$, where $G$ is metacyclic $p$-group and $p>3$. First we show that $H$ is metacyclic. Let $\bar{G}$ and $\bar{H}$ be the factor groups $G / G^{\prime(p)}, H / H^{\prime(p)}$ respectively. Since by Lemma $3\left(\omega\left(F\left[G^{\prime}\right]\right) F[G]\right)^{p}=$ $\omega\left(F\left[G^{\prime(p)}\right]\right) F[G]$ we have

$$
F[\bar{G}] \simeq F[G] /\left(\omega\left(F\left[G^{\prime}\right]\right) F[G]\right)^{p} \simeq F[H] /\left(\omega\left(F\left[H^{\prime}\right]\right) F[H]\right)^{p} \simeq F[\bar{H}] .
$$

The group $\bar{G}$ satisfies the assumption of Lemma 4 , so

$$
\omega_{1}(F[\bar{G}])=\omega\left(F\left[\Omega_{1}(\bar{G})\right]\right) F[\bar{G}] .
$$

By the obvious inclusion $\omega\left(F\left[\Omega_{1}(\bar{H})\right]\right) F[\bar{H}] \subset \omega_{1}(F[\bar{H}])$ we have then

$$
\begin{aligned}
\left|\bar{H} / \Omega_{1}(\bar{H})\right| & =\operatorname{dim}_{F} F\left[\bar{H} / \Omega_{1}(\bar{H})\right]=\operatorname{dim}_{F} F[\bar{H}]-\operatorname{dim}_{F} \omega\left(F\left[\Omega_{1}(\bar{H})\right]\right) F[\bar{H}] \\
& \geq \operatorname{dim}_{F} F[\bar{H}]-\operatorname{dim}_{F} \omega_{1}(F[\bar{H}])=\operatorname{dim}_{F} F[\bar{G}]-\operatorname{dim}_{F} \omega_{1}(F[\bar{G}]) \\
& =\operatorname{dim}_{F} F\left[\bar{G} / \Omega_{1}(\bar{G})\right]=\left|\bar{G} / \Omega_{1}(\bar{G})\right|
\end{aligned}
$$

which implies $\left|\Omega_{1}(\bar{H})\right| \leq\left|\Omega_{1}(\bar{G})\right|=p^{2}$. Hence, since $\bar{H}$ is nonabelian and $p>2$, $\left|\Omega_{1}(\bar{H})\right|=p^{2}$. Now, by [3, III.11.6] $\bar{H}$ is metacyclic. But we have assumed that $p>3$ and from Corollary $1 H^{\prime}$ is cyclic, that is $H^{\prime(p)}=\Phi\left(H^{\prime}\right) \gamma_{3}(H)$. So by $[\mathbf{3}$, III.11.3] $H$ is metacyclic too. The fact that $\bar{H}$ is metacyclic and more, that $\bar{G} \simeq \bar{H}$, one can obtain also from [6, Theorem 6.25].

Now let $G$ be generated by elements $x, y$ with defining relations

$$
x^{p^{m}}=1, \quad y^{p^{n}}=x^{p^{k}}, \quad y^{-1} x y=x^{r}
$$

with suitable integers $m, n, k$ and $r$ [3, III.11.2]. The isomorphism of $G$ and $H$ we prove by induction on the order of $\langle x\rangle \cap\langle y\rangle$. So assume first that the generators $x, y$ of $G$ satisfy

$$
x^{p^{m}}=1, \quad y^{p^{n}}=1, \quad y^{-1} x y=x^{r}
$$


that is $\langle x\rangle \cap\langle y\rangle=1$. We proceed by induction on the order of $G^{\prime}$. The case $\left|G^{\prime}\right|=1$ is known [5, 14.2.7ii]. Since $H$ is metacyclic the isomorphism of $G$ and $H$ for $\left|G^{\prime}\right|=p$ follows immediately from [1, Lemma 4 and Corollary of Theorem 2]. This is also the special case of [6, Theorem 6.25]. Let us assume now that $\left|G^{\prime}\right|=p^{s}$, where $s>1$. We have $\Omega_{1}\left(G^{\prime}\right)=G^{\left(p^{s-1}\right)}$, so by Lemma 3

$$
\begin{aligned}
F\left[G / \Omega_{1}\left(G^{\prime}\right)\right] & \simeq F[G] /\left(\omega\left(F\left[G^{\prime}\right]\right) F[G]\right)^{p^{s-1}} \\
& \simeq F[H] /\left(\omega\left(F\left[H^{\prime}\right]\right) F[H]\right)^{p^{s-1}} \simeq F\left[H / \Omega_{1}\left(H^{\prime}\right)\right]
\end{aligned}
$$

and by the induction $G / \Omega_{1}\left(G^{\prime}\right) \simeq H / \Omega_{1}\left(H^{\prime}\right)$. Since $G / \Omega_{1}\left(G^{\prime}\right)$ is generated by $\bar{x}=x \Omega_{1}\left(G^{\prime}\right), \bar{y}=y \Omega_{1}\left(G^{\prime}\right)$ with relations

$$
\bar{x}^{p^{m-1}}=1, \quad \bar{y}^{p^{n}}=1, \quad \bar{y}^{-1} \overline{x y}=\bar{x}^{r}
$$

there exist generators $u, v$ of $H$ such that

$$
u^{p^{m-1}}, v^{p^{n}} \in \Omega_{1}\left(H^{\prime}\right), \quad v^{-1} u v \equiv u^{r}\left(\bmod \Omega_{1}\left(H^{\prime}\right)\right) .
$$

Let $h \in \Omega_{1}\left(H^{\prime}\right)$ be such an element that $v^{-1} u v=u^{r} h$, and suppose $\Omega_{1}\left(H^{\prime}\right)$ is not a subgroup of $\langle u\rangle$. Then $(u, v)=u^{-1} v^{-1} u v=u^{r-1} h$ is a generator of $H^{\prime}$ and $(u, v)^{p}=\left(u^{r-1} h\right)^{p}=u^{p(r-1)}$. But $H^{\prime}$ is cyclic and $\left|H^{\prime}\right|>p$ so that we have $\Omega_{1}\left(H^{\prime}\right) \leq\left\langle(u, v)^{p}\right\rangle=\left\langle u^{p(r-1)}\right\rangle \leq\langle u\rangle$. A contradiction. Thus $\Omega_{1}\left(H^{\prime}\right)=\left\langle u^{p^{m-1}}\right\rangle$ and $v^{p^{n}} \in\left\langle u^{p^{m-1}}\right\rangle, v^{-1} u v=u^{r+t p^{m-1}}$ for suitable $t$. Suppose that $v^{p^{n}} \neq 1$. If $n>m$, then $\exp (G)=p^{n}<p^{n+1}=\exp (H)$ which by Corollary 2 is impossible. If $n<m$ then one can choose an element $u_{1}$ of $\langle u\rangle$ such that $u_{1}^{p^{n}}=v^{p^{n}}$. Now replacing $v$ by $v_{1}=v u_{1}^{-1}$ we have $H=\left\langle u, v_{1}\right\rangle, v_{1}^{p^{n}}=1$ and $v_{1}^{-1} u v_{1}=v^{-1} u v$. The isomorphism of $G$ and $H$ follows now from [2, Lemma 8].

Suppose now that $x$ and $y$ are generators of $G$ with the defining relations (1), the possible smallest order of $\langle x\rangle \cap\langle y\rangle$ and $\langle x\rangle \cap\langle y\rangle \neq 1$. Remark that the order of $y$ must be greater than the order of $x$. Otherwise, using standard considerations one can replace $y$ by an element $y_{1}$ such that $\left\langle x, y_{1}\right\rangle=G$ and $\langle x\rangle \cap\left\langle y_{1}\right\rangle=1$. Assuming $G / \Omega_{1}\left(G^{\prime}\right) \simeq H / \Omega_{1}\left(H^{\prime}\right)$ as above we can choose generators $u$ and $v$ of $H$ such that $\Omega_{1}\left(H^{\prime}\right) \leq\langle u\rangle$ and

$$
u^{p^{m}}=1, \quad v^{-1} u v=u^{r+t p^{m-1}}, \quad v^{p^{n}}=u^{p^{k}+s p^{m-1}}
$$

for suitable integers $s, t$. If $k<m-1$, then $p^{k}+s p^{m-1} \not \equiv 0\left(\bmod p^{m}\right)$ and there exist an element $u_{1} \in\langle u\rangle$ such that $\langle u\rangle=\left\langle u_{1}\right\rangle, v^{p^{n}}=u_{1}^{p^{k}}$ and $v^{-1} u_{1} v=u_{1}^{r+t p^{m-1}}$. So by [2, Lemma 8] $G \simeq H$. If $p^{k}+s p^{m-1} \equiv 0\left(\bmod p^{m}\right)$, then $v^{p^{n}}=1$ which implies $\exp (H)=p^{n}<\exp G$. By Corollary 2 it is impossible. This completes the proof.

Acknowledgement. I am grateful to Professor E. R. Puczyłowski for his valuable suggestions during the preparation of the paper.

\section{REFERENCES}

1. C. Baginski, Groups of units of modular group algebras, Proc. Amer. Math. Soc. 101 (1987), 619-624.

2. B. G. Basmaji, The isomorphism of two metacyclic groups, Proc. Amer. Math. Soc. 22 (1969), 175-182.

3. B. Huppert, Endliche Gruppen. I, Springer-Verlag, Berlin and New York, 1967. 
4. D. S. Passman, The group algebras of groups of order $p^{4}$ over a modular field, Michigan Math. J. 12 (1965), 405-415.

5. _ The algebraic structure of group rings, Wiley-Interscience, New York, 1978.

6. R. Sandling, The isomorphism problem for group rings: A survey, Lecture Notes in Math., vol. 1142, Springer-Verlag, Berlin and New York, 1985, pp. 256-288.

Institute of Mathematics, University of Warsaw, Bialystok Division, AkaDEMICKA 2, 15-267 BIALYSTOK, POLAND 